\title{
MULTIPLICITY OF SOLUTIONS FOR GENERALIZED QUASILINEAR SCHRÖDINGER EQUATIONS
}

\section{GUOFA LI and BITAO CHENG}

College of Mathematics and Statistics

Qujing Normal University

Qujing, Yunnan 655011

P. R. China

e-mail: liguofa2013@163.com

chengbitao2006@126.com

\begin{abstract}
In this paper, we study the following quasilinear Schrödinger equations:

$-\operatorname{div}\left(g^{2}(u) \nabla u\right)+g(u) g^{\prime}(u)|\nabla u|^{2}+V(x) u=K(x) h(u)+\lambda W(x)|u|^{p-2} u, x \in \mathbb{R}^{N}, \quad$ (P)

where $N \geq 3, V, K \in C\left(\mathbb{R}^{N}, \mathbb{R}^{+}\right)$are given potentials, $1<p<2, \lambda>0$ is a

small parameter, $g$ is a $C^{1}$ even function with $g(0)=1, \lim _{t \rightarrow+\infty} g(t)=a \in(0,1)$

and $g^{\prime}(t) \leq 0$ for all $t \geq 0$, and $h \in C(\mathbb{R}, \mathbb{R})$ satisfies superlinear growth at infinity. We get the existence results of multiplicity of nontrivial solutions for problem $(\mathrm{P})$.
\end{abstract}

2010 Mathematics Subject Classification: 35J20, 35J62.

Keywords and phrases: quasilinear Schrödinger equations, multiplicity of solutions, Mountain pass theorem, vanishing potentials.

Received July 17, 2020

(C) 2020 Scientific Advances Publishers 


\section{Introduction}

In the last few years, there are many papers have studied the following quasilinear Schrödinger equations:

$$
i z_{t}=-\Delta z+W(x) z-\eta\left(x,|z|^{2}\right) z+\kappa\left[\Delta\left(\varphi\left(|z|^{2}\right) \varphi^{\prime}\left(|z|^{2}\right)\right] z, \quad x \in \mathbb{R}^{N}\right.
$$

where $z: \mathbb{R} \times \mathbb{R}^{N} \rightarrow \mathbb{C}, W: \mathbb{R}^{N} \rightarrow \mathbb{R}$ is a given potential, $\kappa$ is a parameter and $\eta: \mathbb{R}^{N} \times \mathbb{R}^{+} \rightarrow \mathbb{R}, \varphi: \mathbb{R}^{+} \rightarrow \mathbb{R}$ are real functions. Quasilinear equations of the form (1.1) have been derived as mathematical models of several physical phenomena corresponding to various types of the nonlinear terms $\varphi$ and $\eta$. For example, if $\varphi(s)=s$

and $\eta(s)=-\alpha-\frac{\beta}{(a+s)^{3}}$ for some positive constants $\alpha$ and $\beta$, then (1.1) become the basic equation to study the oscillations of a superfluid film, for more details on the physical backgrounds, we refer the readers to $[9,11,21]$ and references therein. The nonlinear Schrödinger equations describe the propagation of optical pulses with higher-dispersion. These solitons play an important and key role for information transfer via optical fibers [37]. Our interests in the present paper are existence of standing wave solutions for (1.1), that is, solutions of the form $z(t, x)=\exp (-i E t) u(x)$, where $E \in \mathbb{R}$ and $u$ is a real function. Submitting $z(t, x)=\exp (-i E t) u(x)$ into (1.1), we get

$$
-\Delta u+V(x) u+\kappa\left[\Delta\left(\varphi\left(|u|^{2}\right)\right) \varphi^{\prime}\left(|u|^{2}\right) u=\eta\left(x, u^{2}\right) u, \quad x \in \mathbb{R}^{N},\right.
$$

where $V(x):=W(x)-E$. In particular, setting $l(x, t):=\eta\left(x, t^{2}\right) t$, then if $\varphi(t)=t$ and $\varphi(t)=\sqrt{1+t},(1.2)$ turns into

$$
-\Delta u+V(x) u+\kappa\left[\Delta|u|^{2}\right] u=l(x, u), \quad x \in \mathbb{R}^{N},
$$


and

$$
-\Delta u+V(x) u+\kappa\left[\Delta\left(1+u^{2}\right)^{1 / 2}\right] \frac{u}{2\left(1+u^{2}\right)^{1 / 2}}=l(x, u), \quad x \in \mathbb{R}^{N} .
$$

If $\kappa=0$, problems (1.3) and (1.4) are semilinear Schrödinger equations, which have been studied extensively via variational methods in past thirty years, see, e.g., [8, 23, 32] and references therein.

If $\kappa<0$, a lot of existence and multiplicity of weak solutions for (1.3) have been obtained by many papers via variational methods, see, e.g., $[1,12,13,17,27,38,40,42,51,53,54]$, where there are three main arguments used in the above references, one is the constrained minimization argument, namely consider a minimization on a convenient constraint to get existence results, see [27, 38, 40, 51]. The second is perturbed argument, namely add a high order perturbation term such that the perturbed function is smooth, see [33, 34, 35, 49, 50]. The third is to make a change of variable (dual approach $v=f^{-1}(u)$ with $f$ satisfies $f^{\prime}(t)=1 / \sqrt{1-\kappa f^{2}(t)}$ for $t \in[0,+\infty)$ and $f(t)=-f(-t)$ for $\left.t \in(-\infty, 0]\right)$ to reduce (1.3) into a semilinear equation like the case of $\kappa=0$, see [12, 17, 42]. Moreover, a lot of existence and multiplicity of weak solutions results for (1.4) have been obtained by many authors via variational methods with the change of variable $v=f^{-1}(u)$, see, e.g., [14, 15, 16, 28, 44, 45, 46, 47].

More recently, there are many papers have studied existence results of nontrivial solutions for the following generalized quasilinear Schrödinger equations, see [30, 31, 45, 47, 48]

$$
-\operatorname{div}\left(g^{2}(u) \nabla u\right)+g(u) g^{\prime}(u)|\nabla u|^{2}+V(x) u=l(x, u), x \in \mathbb{R}^{N},
$$

where $g \in C^{1}\left(\mathbb{R}, \mathbb{R}^{+}\right)$satisfies

(G) $g$ is a even function with $g(0)=1$ and $g^{\prime}(t) \geq 0$ for all $t \geq 0$. 
In these papers, a change of variable was used to reduced the problem (1.5) to semilinear problem. In fact, their results focused on the general function $\varphi\left(|u|^{2}\right)$ in (1.2). Obviously, the change of variable $v=f^{-1}(u)$ satisfies condition $(\mathrm{G})$.

If $\kappa>0$ is small enough, by using a change of variables, namely, $s=G^{-1}(t)$ for $t \in[0,+\infty)$ and $G^{-1}(t)=-G^{-1}(-t)$ for $t \in(-\infty, 0)$, where the inverse of $G^{-1}(t)$ is

$$
\begin{gathered}
G(s)=\int_{0}^{s} g(t) d t, \\
g(t)= \begin{cases}\sqrt{1-\kappa t^{2}}, & 0 \leq t<\sqrt{\frac{1}{3 \kappa}} \\
\frac{1}{3 \sqrt{2 \kappa} t}+\sqrt{\frac{1}{6}}, & t \geq \sqrt{\frac{1}{3 \kappa}}, \\
g(-t), & t<0,\end{cases}
\end{gathered}
$$

the authors in $[2,4,41,43,53]$ reduced (1.3) to a semilinear one, and an Orlicz space framework was used. The same change of variable was also used in [52]. Soon after, Huang and Jia get the existence of positive solutions for (1.3) with $\kappa=1$ by using same change of variable, see [22]. Moreover, if $0<\kappa<2$, Shen and Wang have studied the existence of nontrivial solutions for (1.4) by using the change of variable (1.6), where $g(t)=\sqrt{1-\left(\kappa t^{2}\right) /\left[2\left(1+t^{2}\right)\right]}$, see [44].

In order to study problem (1.2) with the general functions $\varphi\left(|u|^{2}\right)$, we concerned the existence of positive solutions for (1.5) under the conditions (GD) and (V), see [25, 26].

(GD) $g$ is a even function with $g(0)=1, \lim _{t \rightarrow+\infty} g(t)=a \in(0,1)$ and $g^{\prime}(t) \leq 0$ for all $t \geq 0$. 


$$
\text { (V) } 0<V_{0}=\inf _{x \in \mathbb{R}^{N}} V(x), V(x) \leq V_{\infty}:=\lim _{|x| \rightarrow \infty} V(x)<\infty .
$$

In fact, we say the potential $V(x)$ is the case of well potential if $V(x)$ satisfies condition (V). Obviously, there are many functions satisfy condition (GD). For example, $g(t)$ is defined in (1.7) with $a=\sqrt{1 / 6}$ and $g(t)=\sqrt{1-\left(\kappa t^{2}\right) /\left[2\left(1+t^{2}\right)\right]} \quad$ with $\quad a=\sqrt{1-\kappa / 2}$, where $0<\kappa<2$. However, as far as we know in the literature there is no result of existence of nontrivial solutions for (1.5). In the present paper, we try to fill this gap and give an affirmative answer to this question. We consider problem (1.5) with $l(x, u)=K(x) h(u)+\lambda W(x)|u|^{p-2} u$, that is the following generalized quasilinear Schrödinger equations:

$$
\begin{gathered}
-\operatorname{div}\left(g^{2}(u) \nabla u\right)+g(u) g^{\prime}(u)|\nabla u|^{2}+V(x) u=K(x) h(u)+\lambda W(x)|u|^{p-2} u, \\
x \in \mathbb{R}^{N},
\end{gathered}
$$

where $N \geq 3,1<p<2, \lambda>0$ is a small parameter, $W \in L^{\frac{2}{2-p}}\left(\mathbb{R}^{N}, \mathbb{R}^{+}\right)$, $h \in C(\mathbb{R}, \mathbb{R})$ satisfies superlinear growth at infinity, $g$ is of $C^{1}\left(\mathbb{R}, \mathbb{R}^{+}\right)$ satisfies (GD), and $V(x), K(x)$ are positive continuous functions and $(V, K) \in \mathcal{K}$, that is, $V$ and $K$ satisfy the following conditions:

$$
\begin{aligned}
& \left(\mathrm{V}_{0}\right) V(x)>0, \forall x \in \mathbb{R}^{N} \text { and } \lim _{|x| \rightarrow \infty} V(x)=0 . \\
& \left(\mathrm{K}_{1}\right)(\mathrm{i}) K(x)>0, \forall x \in \mathbb{R}^{N}, K \in L^{\infty}\left(\mathbb{R}^{N}\right) .
\end{aligned}
$$

(ii) if $\left\{A_{n}\right\} \subset \mathbb{R}^{N}$ is a sequence of Borel sets such that $\left|A_{n}\right| \leq R$ for all $n$ and some $R>0$, then

$$
\lim _{r \rightarrow+\infty} \int_{A_{n} \cap B_{r}^{c}(0)} K(x) d x=0 .
$$


$\left(\mathrm{K}_{2}\right) \frac{K}{V} \in L^{\infty}\left(\mathbb{R}^{N}\right)$.

$\left(\mathrm{K}_{3}\right)$ There is $\sigma \in\left(2,2^{*}\right)$ such that $\lim _{|x| \rightarrow \infty} \frac{K(x)}{[V(x)]_{2^{*}-2}^{\frac{2^{*}-\sigma}{*}}}=0$.

For the case of potential $V(x)$ vanishes at infinity, there are many papers have shown existence of solutions for Schrödinger equations (see $[5,6,10,24,29,55,56])$ and quasilinear Schrödinger equations (see [1, 2, 19, 20, 36, 48] and references therein). In [5], Ambrosetti et al. considered the following Schrödinger equation:

$$
-\varepsilon^{2} \Delta u+V(x) u=K(x)|u|^{p}, x \in \mathbb{R}^{N},
$$

where $N \geq 3, V, K: \mathbb{R}^{N} \rightarrow \mathbb{R}$ are smooth functions and there exist constants $c_{1}, c_{2}, c_{3}, \alpha_{1}, \alpha_{2}>0$ such that

$$
(\mathrm{VK}) \frac{c_{3}}{1+|x|^{c_{1}}} \leq V(x) \leq \alpha_{1} \text { and } 0<K(x) \leq \frac{\alpha_{2}}{1+|x|^{c_{2}}}, \forall x \in \mathbb{R}^{N}
$$

Moreover, $c_{1}, c_{2}$ satisfy

$$
\frac{N+2}{N-2}-\frac{4 c_{2}}{c_{1}(N-2)}<p \text { if } 0<c_{2}<c_{1} \text { or } p>1 \text {, where } c_{2}>c_{1} \text {. }
$$

Later, in [7], Ambrosetti and Wang considered the condition (VK), but the condition on $V$ was assumed only outside of a ball centered at origin. Moreover, in [3], Alves and Souto considered a more general condition on $V$ and $K$. Precisely, they assumed that $(V, K) \in \mathcal{K}$, they got existence of positive ground state solution for the following equation:

$$
-\Delta u+V(x) u=K(x) h(u), x \in \mathbb{R}^{N} .
$$


In the present paper, we consider the case $(V, K) \in \mathcal{K}$. To state our main result, we assume that $h$ satisfies

(h1) $\lim _{|t| \rightarrow 0} \frac{h(t)}{|t|}=0$ if $\left(\mathrm{K}_{2}\right)$ holds; $\lim _{|t| \rightarrow 0} \frac{h(t)}{|t|^{q-1}}<+\infty$ if $\left(\mathrm{K}_{3}\right)$ holds, where $2<q<2^{*}$.

(h2) There exists $C>0$ such that $|h(t)| \leq C\left(1+|t|^{q-1}\right)$ for all $t \in \mathbb{R}$.

(h3) There exists $\theta>2$ such that $0 \leq \theta H(t) \leq h(t) t$ for all $t \in \mathbb{R}$, where $H(t)=\int_{0}^{t} h(s) d s$.

(h4) $h(t)=-h(-t)$ for all $t \in \mathbb{R}$.

Now, we state our main results as follows:

Theorem 1.1. Assume that (h1)-(h3) hold, $(V, K) \in \mathcal{K} \quad$ and $W \in L^{\frac{2}{2-p}}\left(\mathbb{R}^{N}, \mathbb{R}^{+}\right), 1<p<2$, then there exists a constant $\lambda_{0}>0$ such that for all $\lambda \in\left(0, \lambda_{0}\right)$, problem (1.8) has at least two nontrivial solutions.

Theorem 1.2. Assume that (h1)-(h4) hold, $(V, K) \in \mathcal{K}$ and $W \in L^{\frac{2}{2-p}}\left(\mathbb{R}^{N}, \mathbb{R}^{+}\right), 1<p<2$, then problem (1.8) has infinity many nontrivial solutions.

This paper is organized as follows: in Section 2, we will provide a useful lemma and present some embedding results. In Section 3, we will prove Theorems 1.1 and 1.2. Throughout this paper, we will denote the following notations: $\|u\|_{p}(1<p \leq \infty)$ is the norm in $L^{p}\left(\mathbb{R}^{N}\right) ; \rightarrow$ and $\rightarrow$ denote strong and weak convergence, respectively; $\langle\cdot, \cdot\rangle$ denotes the duality pairing between a Banach space and its dual space; $o_{n}(1)$ denotes $o_{n}(1) \rightarrow 0$ as $n \rightarrow \infty$ and $B_{R}(0)$ denotes a ball centered at the origin with radius $R>0$. 


\section{Preliminaries}

We introduce the subspace

$$
H=\left\{u \in H^{1}\left(\mathbb{R}^{N}\right): \int_{\mathbb{R}^{N}} V(x) u^{2} d x<+\infty\right\},
$$

which is a Hilbert space equipped with the inner product

$$
\langle u, v\rangle=\int_{\mathbb{R}^{N}}(\nabla u \cdot \nabla v+V(x) u v) d x, u, v \in H
$$

and the norm

$$
\|u\|=\left[\int_{\mathbb{R}^{N}}\left(|\nabla u|^{2}+V(x) u^{2}\right) d x\right]^{1 / 2}
$$

Denote by $L_{K}^{r}\left(\mathbb{R}^{N}\right)$ the weighted Lebesgue function space

$$
L_{K}^{r}\left(\mathbb{R}^{N}\right)=\left\{u \in H^{1}\left(\mathbb{R}^{N}\right): u \text { is measurable and } \int_{\mathbb{R}^{N}} K(x)|u|^{r} d x<+\infty\right\},
$$

and owe with the norm

$$
\|u\|_{r, K}=\left(\int_{\mathbb{R}^{N}} K(x)|u|^{r} d x\right)^{1 / r}, u \in H .
$$

The natural energy functional corresponding to problem (1.8) is given by

$$
\begin{array}{r}
I(u)=\frac{1}{2} \int_{\mathbb{R}^{N}} g^{2}(u)|\nabla u|^{2} d x+\frac{1}{2} \int_{\mathbb{R}^{N}} V(x)|u|^{2} d x-\int_{\mathbb{R}^{N}} K(x) H(u) d x \\
\quad-\frac{\lambda}{p} \int_{\mathbb{R}^{N}} W(x)|u|^{p} d x,
\end{array}
$$


where $H(t)=\int_{0}^{t} h(s) d s$. By making the following change of variable

$$
v=G(u)=\int_{0}^{u} g(s) d s
$$

the functional $I(u)$ can be written by

$$
\begin{aligned}
J(v)=\frac{1}{2} \int_{\mathbb{R}^{N}}|\nabla v|^{2} d x+\frac{1}{2} \int_{\mathbb{R}^{N}} V(x)\left|G^{-1}(v)\right|^{2} d x- & \int_{\mathbb{R}^{N}} K(x) H\left(G^{-1}(v)\right) d x \\
& -\frac{\lambda}{p} \int_{\mathbb{R}^{N}} W(x)\left|G^{-1}(v)\right|^{p} d x .
\end{aligned}
$$

$J(v)$ is the variational functional of the following equation:

$$
\begin{gathered}
-\Delta v+V(x) \frac{G^{-1}(v)}{g\left(G^{-1}(v)\right)}=K(x) \frac{h\left(G^{-1}(v)\right)}{g\left(G^{-1}(v)\right)}+\lambda W(x) \frac{\left|G^{-1}(v)\right|^{p-2} G^{-1}(v)}{g\left(G^{-1}(v)\right)}, \\
x \in \mathbb{R}^{N}
\end{gathered}
$$

where $G^{-1}(v)$ is the inverse function of $G(u)$. Next, we will establish the smoothness of $J$. First, we give some properties of $g(t)$ and $G(t)$.

Lemma 2.1 ([25], Lemma 2.1). The functions $g(t), G(t)=\int_{0}^{t} g(s) d s$ enjoy the following properties under the condition (GD)

(1) $G(t)$ is invertible, $G(t)$ and the inverse $G^{-1}(t)$ are odd.

(2) $\frac{t}{g(t)} g^{\prime}(t) \leq 0$ for all $t \geq 0$.

(3) $|t| \leq\left|G^{-1}(t)\right| \leq \frac{|t|}{a}$ for all $t \in \mathbb{R}$. 
(4) $\frac{G^{-1}(t)}{t}$ is nondecreasing for all $t \in \mathbb{R}$ and $\lim _{t \rightarrow 0} \frac{G^{-1}(t)}{t}=1$, $\lim _{t \rightarrow \infty} \frac{G^{-1}(t)}{t}=\frac{1}{a}$.

(5) $t^{2} \leq \frac{t}{g(t)} G(t) \leq \frac{t^{2}}{a}$ for all $t \in \mathbb{R}$.

Lemma 2.2. $J$ is well defined in $H$ and $J \in C^{1}(H, \mathbb{R})$. Moreover,

$$
\begin{aligned}
&\left\langle J^{\prime}(v), \psi\right\rangle= \int_{\mathbb{R}^{N}} \nabla v \nabla \psi d x+\int_{\mathbb{R}^{N}} V(x) \frac{G^{-1}(v)}{g\left(G^{-1}(v)\right)} \psi d x \\
&-\int_{\mathbb{R}^{N}} K(x) \frac{h\left(G^{-1}(v)\right)}{g\left(G^{-1}(v)\right)} \psi d x-\lambda \int_{\mathbb{R}^{N}} W(x) \frac{\left|G^{-1}(v)\right|^{p-2} G^{-1}(v)}{g\left(G^{-1}(v)\right)} \psi d x, \\
& \forall \psi \in H .
\end{aligned}
$$

Proof. It deduces from Lemma 2.1(3) that

$$
\int_{\mathbb{R}^{N}} V(x)\left|G^{-1}(v)\right|^{2} d x \leq \frac{1}{a^{2}} \int_{\mathbb{R}^{N}} V(x)|v|^{2} d x, \forall v \in H .
$$

By (h1) and (h2), for any $\varepsilon>0$, there exists $C_{\varepsilon}>0$ such that

$$
|h(t)| \leq \varepsilon|t|+C_{\varepsilon}|t|^{q-1}, \forall t \in \mathbb{R},
$$

where $2<q<2^{*}$. Then

$$
|H(t)| \leq \frac{\varepsilon}{2}|t|^{2}+\frac{C_{\varepsilon}}{q}|t|^{q}, \forall t \in \mathbb{R} .
$$

Hence,

$$
\int_{\mathbb{R}^{N}} K(x)\left|H\left(G^{-1}(v)\right)\right| d x \leq \int_{\mathbb{R}^{N}} K(x)\left[\frac{\varepsilon}{2 a^{2}}|v|^{2}+\frac{C_{\varepsilon}}{q a^{q}}|v|^{q}\right] d x<+\infty, \forall v \in H .
$$


Since $W \in L^{\frac{2}{2-p}}\left(\mathbb{R}^{N}, \mathbb{R}^{+}\right), 1<p<2$, by the Hölder inequality, we have

$$
\begin{aligned}
\int_{\mathbb{R}^{N}} W(x)\left|G^{-1}(v)\right|^{p} d x & \leq \frac{1}{a^{p}} \int_{\mathbb{R}^{N}} W(x)|v|^{p} d x \\
& \leq \frac{1}{a^{p}}\left[\int_{\mathbb{R}^{N}}|W(x)|^{\frac{2}{2-p}} d x\right]^{\frac{2-p}{2}}\left[\int_{\mathbb{R}^{N}}|v|^{2} d x\right]^{\frac{p}{2}}<+\infty, \forall v \in H .
\end{aligned}
$$

Thus, $J$ is well defined.

Next, we verify that $J \in C^{1}(H, \mathbb{R})$. Note that for any $v, \psi \in H$ is fixed, given $0<t<1$, by the mean value theorem, there exists $\epsilon \in(0,1)$ such that

$$
\begin{aligned}
\left|\frac{\left|G^{-1}(v+t \psi)\right|^{2}-\left|G^{-1}(v)\right|^{2}}{t}\right| & =2\left|\frac{G^{-1}(v+\epsilon t \psi)}{g\left(G^{-1}(v+\epsilon t \psi)\right)} \psi\right| \\
& \leq \frac{2}{a^{2}}|(v+\epsilon t \psi) \psi| \\
& \leq \frac{2}{a^{2}}(|v|+|\psi|)|\psi| \in L^{1}\left(\mathbb{R}^{N}\right) .
\end{aligned}
$$

Then by the Lebesgue dominated convergence theorem, we conclude that

$$
\lim _{t \rightarrow 0} \frac{1}{2} \int_{\mathbb{R}^{N}} V(x) \frac{\left|G^{-1}(v+t \psi)\right|^{2}-\left|G^{-1}(v)\right|^{2}}{t} d x=\int_{\mathbb{R}^{N}} V(x) \frac{G^{-1}(v)}{g\left(G^{-1}(v)\right)} \psi d x .
$$

Similarly, by (2.2), we have

$$
\lim _{t \rightarrow 0} \int_{\mathbb{R}^{N}} K(x) \frac{H\left(G^{-1}(v+t \psi)\right)-H\left(G^{-1}(v)\right)}{t} d x=\int_{\mathbb{R}^{N}} K(x) \frac{h\left(G^{-1}(v)\right)}{g\left(G^{-1}(v)\right)} \psi d x .
$$


Moreover, for any $v, \psi \in H$ are fixed, given $0<t<1$, by the mean value theorem, there exists $\mu \in(0,1)$ such that

$$
\begin{aligned}
\left|\frac{\left|G^{-1}(v+t \psi)\right|^{p}-\left|G^{-1}(v)\right|^{p}}{t}\right| & =p \frac{\left|G^{-1}(v+\mu t \psi)\right|^{p-1}}{g\left(G^{-1}(v+\mu t \psi)\right)}|\psi| \\
& \leq \frac{p}{a^{p}}|v+\mu t \psi|^{p-1}|\psi| .
\end{aligned}
$$

Since $\frac{2-p}{2}+\frac{p-1}{2}+\frac{1}{2}=1, W \in L^{\frac{2}{2-p}}\left(\mathbb{R}^{N}, \mathbb{R}^{+}\right), 1<p<2, \quad$ by the Hölder inequality and Minkowski inequality, we get

$$
\begin{aligned}
\int_{\mathbb{R}^{N}} W(x)|v+\mu t \psi|^{p-1}|\psi| d x \\
\leq\left[\int_{\mathbb{R}^{N}}|W(x)|^{\frac{2}{2-p}} d x\right]^{\frac{2-p}{2}}\left[\int_{\mathbb{R}^{N}}|v+\mu t \psi|^{2} d x\right]^{\frac{p-1}{2}}\left[\int_{\mathbb{R}^{N}}|\psi|^{2} d x\right]^{\frac{1}{2}} \\
\leq\left[\int_{\mathbb{R}^{N}} \mid W(x) \frac{2}{2-p} d x\right]^{\frac{2-p}{2}}\left[\left(\int_{\mathbb{R}^{N}}|v|^{2} d x\right)^{\frac{1}{2}}+\left[\int_{\mathbb{R}^{N}}|\psi|^{2} d x\right)^{\frac{1}{2}}\right]^{p-1}\left[\int_{\mathbb{R}^{N}}|\psi|^{2} d x\right]^{\frac{1}{2}} \\
\quad<+\infty .
\end{aligned}
$$

Hence, by the Lebesgue dominated convergence theorem, we have

$$
\lim _{t \rightarrow 0} \frac{\lambda}{p} \int_{\mathbb{R}^{N}} W(x) \frac{\left|G^{-1}(v+t \psi)\right|^{p}-\left|G^{-1}(v)\right|^{p}}{t} d x=\lambda \int_{\mathbb{R}^{N}} W(x) \frac{\left|G^{-1}(v)\right|^{p-2} G^{-1}(v)}{g\left(G^{-1}(v)\right)} \psi d x .
$$


This means that $J \in C^{1}(H, \mathbb{R})$ and

$$
\begin{aligned}
&\left\langle J^{\prime}(v), \psi\right\rangle= \int_{\mathbb{R}^{N}} \nabla v \nabla \psi d x+\int_{\mathbb{R}^{N}} V(x) \frac{G^{-1}(v)}{g\left(G^{-1}(v)\right)} \psi d x \\
&-\int_{\mathbb{R}^{N}} K(x) \frac{h\left(G^{-1}(v)\right)}{g\left(G^{-1}(v)\right)} \psi d x-\lambda \int_{\mathbb{R}^{N}} W(x) \frac{\left|G^{-1}(v)\right|^{p-2} G^{-1}(v)}{g\left(G^{-1}(v)\right)} \psi d x, \\
& \forall \psi \in H .
\end{aligned}
$$

Second, we show relations with critical point of $J$ and weak solution of problem (1.8).

Lemma 2.3. Assume that $\left(\mathrm{V}_{0}\right)$ and (h1)-(h2) hold. If $v \in H$ is a critical point of $J$, then $u=G^{-1}(v)$ is a weak solution of problem (1.8).

Proof. Since $v$ is a critical point of $J$, we have

$$
\begin{aligned}
\int_{\mathbb{R}^{N}} \nabla v \nabla \psi d x+\int_{\mathbb{R}^{N}} V(x) \frac{G^{-1}(v)}{g\left(G^{-1}(v)\right)} \psi d x \\
=\int_{\mathbb{R}^{N}} K(x) \frac{h\left(G^{-1}(v)\right)}{g\left(G^{-1}(v)\right)} \psi d x+\lambda \int_{\mathbb{R}^{N}} W(x) \frac{\left|G^{-1}(v)\right|^{p-2} G^{-1}(v)}{g\left(G^{-1}(v)\right)} \psi d x,
\end{aligned}
$$

for all $\psi \in H$.

Since $v \in H$, it deduces from $\left(\mathrm{V}_{0}\right)$ and Lemma 2.1 (3) that $u:=G^{-1}(v) \in H$. For each $\varphi \in C_{0}^{\infty}\left(\mathbb{R}^{N}\right)$, taking $\psi:=g(u) \varphi \in H$ in (2.3), we have

$$
\begin{aligned}
\int_{\mathbb{R}^{N}} \nabla v\left(g^{\prime}(u) \varphi \nabla u+g(u) \nabla \varphi\right) d x+\int_{\mathbb{R}^{N}} V(x) \frac{u}{g(u)} g(u) \varphi d x \\
=\int_{\mathbb{R}^{N}} \frac{h(u)}{g(u)} g(u) \varphi d x+\lambda \int_{\mathbb{R}^{N}} W(x) \frac{|u|^{p-2} u}{g(u)} g(u) \varphi d x .
\end{aligned}
$$


Note that $v=G(u)$ and $\nabla v=g(u) \nabla u$, then

$$
\begin{aligned}
\int_{\mathbb{R}^{N}}\left[g^{2}(u) \nabla u \nabla \varphi+g(u) g^{\prime}(u)|\nabla u|^{2} \varphi\right] d x+\int_{\mathbb{R}^{N}} V(x) u \varphi d x \\
=\int_{\mathbb{R}^{N}} h(u) \varphi d x+\lambda \int_{\mathbb{R}^{N}} W(x)|u|^{p-2} u \varphi d x,
\end{aligned}
$$

for $\varphi \in C_{0}^{\infty}\left(\mathbb{R}^{N}\right)$. Therefore, $u$ is a weak solution of problem (1.8).

By $\left(\mathrm{V}_{0}\right)$, we have the following embedding theorems.

Lemma 2.4 ([18], Lemma 2.1). Assume that $(V, K) \in \mathcal{K}$, then $H \hookrightarrow L_{K}^{r}\left(\mathbb{R}^{N}\right)$ is continuous for all $r \in\left[2,2^{*}\right]$ if $\left(\mathrm{K}_{2}\right)$ holds; $H \hookrightarrow L_{K}^{\sigma}\left(\mathbb{R}^{N}\right)$ is continuous for all $\sigma \in\left(2,2^{*}\right)$ if $\left(\mathrm{K}_{3}\right)$ holds.

It deduces from Lemma 2.4 that there exists a constant $\gamma_{r}>0$ which is dependent of $r$ such that

$$
\|u\|_{r, K} \leq \gamma_{r}\|u\| \text { for all } r \in\left[2,2^{*}\right]
$$

Lemma 2.5 ([3], Proposition 2.1). Assume that $(V, K) \in \mathcal{K}$, then $H \hookrightarrow L_{K}^{q}\left(\mathbb{R}^{N}\right)$ is compact for all $q \in\left(2,2^{*}\right)$ if $\left(\mathrm{K}_{2}\right)$ holds; $H \hookrightarrow L_{K}^{\sigma}\left(\mathbb{R}^{N}\right)$ is compact for all $\sigma \in\left(2,2^{*}\right)$ if $\left(\mathrm{K}_{3}\right)$ holds.

\section{Proof of Theorems 1.1 and 1.2}

First, we discuss $J$ satisfies the Mountain Pass geometry.

Lemma 3.1. Assume that h satisfies (h1) and (h2), then

(1) there exist $\rho, \alpha>0$ such that $J(v) \geq \alpha,\|v\|=\rho$.

(2) there is $v \in H \backslash\{0\}$ such that $J(v)<0$. 
Proof. (1) Set $S_{\rho}=\{v \in H:\|v\|=\rho\}$, combining Lemma 2.1(3), (2.4)

and (2.2), let $\varepsilon=\frac{a^{2}}{2 \gamma_{2}^{2}}$, we have

$$
\begin{aligned}
J(v) \geq & \frac{1}{2} \int_{\mathbb{R}^{N}}|\nabla v|^{2}+\frac{1}{2} \int_{\mathbb{R}^{N}} V(x)|v|^{2} d x-\frac{\lambda}{p a^{p}} \int_{\mathbb{R}^{N}} W(x)|v|^{p} d x \\
& -\frac{\varepsilon}{2 a^{2}} \int_{\mathbb{R}^{N}} K(x)|v|^{2} d x-\frac{C_{\varepsilon}}{q a^{q}} \int_{\mathbb{R}^{N}} K(x)|v|^{q} d x \\
\geq & \frac{1}{2}\|v\|^{2}-\frac{\lambda}{p a^{p}}\|W(x)\|_{2} \frac{2}{2-p}\|v\|^{p}-\frac{\varepsilon \gamma_{2}^{2}}{2 a^{2}}\|v\|^{2}-\frac{C_{\varepsilon}}{q a^{q}} \gamma_{q}^{q}\|v\|^{q} \\
= & \|v\|^{p}\left[\frac{1}{4}\|v\|^{2-p}-\frac{C_{\varepsilon}}{q a^{q}} \gamma_{q}^{q}\|v\|^{q-p}-\frac{\lambda}{p a^{p}}\|W(x)\|_{\frac{2}{2-p}}\right] .
\end{aligned}
$$

Denote

$$
\eta(t)=\frac{1}{4} t^{2-p}-\frac{C_{\varepsilon}}{q a^{q}} \gamma_{q}^{q} t^{q-p}-\frac{\lambda}{p a^{p}}\|W(x)\|_{\frac{2}{2-p}}, t \geq 0
$$

hence,

$$
\eta^{\prime}(t)=\frac{2-p}{4} t^{1-p}-\frac{C_{\varepsilon}}{q a^{q}} \gamma_{q}^{q}(q-p) t^{q-p-1}, t \geq 0
$$

Then $\eta(t)$ arrives its maximum at $t=\left[\frac{(2-p) q}{4 C_{\varepsilon} a^{q} \gamma_{q}^{q}(q-p)}\right]^{\frac{1}{q-2}}$. That is, there is $\rho>0$ such that

$$
\max _{t \geq 0} \eta(t)=\eta(\rho)=\frac{q-2}{4(q-p)}\left[\frac{(2-p) q}{4 C_{\varepsilon} a^{q} \gamma_{q}^{q}(q-p)}\right]^{\frac{2-p}{q-2}}
$$


Therefore, there exist constants $\lambda_{0}$ and $\alpha$ such that $\left.J(v)\right|_{S_{\rho}} \geq \alpha>0$ for all $\lambda \in\left(0, \lambda_{0}\right)$.

(2) It deduces from (h3) that

$$
\lim _{t \rightarrow+\infty} \frac{H(t)}{t^{2}}=+\infty
$$

By Lemma 2.1(3), we have

$$
\begin{aligned}
& \frac{J(t \varphi)}{t^{2}} \leq \frac{1}{2} \int_{\mathbb{R}^{N}}|\nabla \varphi|^{2} d x+\frac{1}{2 a^{2}} \int_{\mathbb{R}^{N}} V(x)|\varphi|^{2} d x-\frac{\lambda}{p} \int_{\mathbb{R}^{N}} W(x) \frac{|t \varphi|^{p}}{t^{2}} d x \\
&-\int_{\mathbb{R}^{N}} K(x) \frac{H\left(G^{-1}(t \varphi)\right)}{(t \varphi)^{2}} \varphi^{2} d x \\
& \leq \frac{1}{2} \int_{\mathbb{R}^{N}}|\nabla \varphi|^{2} d x+\frac{1}{2 a^{2}} \int_{\mathbb{R}^{N}} V(x)|\varphi|^{2} d x-\frac{\lambda}{p} \int_{\mathbb{R}^{N}} W(x) \frac{|t \varphi|^{p}}{t^{2}} d x \\
&-\int_{\mathbb{R}^{N}} K(x) \frac{H\left(G^{-1}(t \varphi)\right)}{\left(G^{-1}(t \varphi)\right)^{2}} \varphi^{2} d x \\
& \rightarrow-\infty, t \rightarrow+\infty,
\end{aligned}
$$

therefore, there exists large enough $t_{0}>0$, let $v=t_{0} \varphi$ with $\|v\|>\rho$ such that $J(v)<0$.

By Lemma 3.1, $J$ satisfies the Mountain Pass geometry. Then there is a $(P S)$ sequence $\left\{v_{n}\right\} \in H$ at level $c$ such that

$$
J\left(v_{n}\right) \rightarrow c, J^{\prime}\left(v_{n}\right) \rightarrow 0 \text { as } n \rightarrow \infty
$$

where

$$
c=\inf _{\gamma \in \Gamma} \sup _{t \in[0,1]} J(\gamma), \Gamma=\{\gamma \in C([0,1], H): \gamma(0)=0, \gamma(1) \neq 0, J(\gamma(1))<0\} .
$$


Second, we establish the boundedness of $(P S)_{c}$ sequence $\left\{v_{n}\right\}$ which is defined in (3.2).

Lemma 3.2. Suppose that $h$ satisfies (h1)-(h3) and $(V, K) \in \mathcal{K}$, then any $(P S)_{c}$ sequence $\left\{v_{n}\right\}$ is bounded in $H$.

Proof. By (3.2), (2) and (3) of Lemma 2.1, we know that

$$
\begin{aligned}
c+1+o_{n}(1) & \left\|v_{n}\right\| \geq J\left(v_{n}\right)-\frac{1}{\theta}\left\langle J^{\prime}\left(v_{n}\right), G^{-1}\left(v_{n}\right) g\left(G^{-1}\left(v_{n}\right)\right)\right\rangle \\
= & \left(\frac{1}{2}-\frac{1}{\theta}\right) \int_{\mathbb{R}^{N}}|\nabla v|^{2} d x-\frac{1}{\theta} \int_{\mathbb{R}^{N}} \frac{G^{-1}\left(v_{n}\right)}{g\left(G^{-1}\left(v_{n}\right)\right)} g^{\prime}\left(G^{-1}\left(v_{n}\right)\right)\left|\nabla v_{n}\right|^{2} d x \\
& +\left(\frac{1}{2}-\frac{1}{\theta}\right) \int_{\mathbb{R}^{N}} V(x)\left|G^{-1}\left(v_{n}\right)\right|^{2} d x \\
& +\int_{\mathbb{R}^{N}} K(x)\left[\frac{1}{\theta} h\left(G^{-1}\left(v_{n}\right)\right) G^{-1}\left(v_{n}\right)-H\left(G^{-1}\left(v_{n}\right)\right)\right] d x \\
& +\left(\frac{1}{\theta}-\frac{1}{p}\right) \lambda \int_{\mathbb{R}^{N}} W(x)\left|G^{-1}\left(v_{n}\right)\right|^{p} d x .
\end{aligned}
$$

Hence, by (h3), we have

$$
\begin{aligned}
c+1+ & o_{n}(1)\left\|v_{n}\right\|+\left(\frac{1}{p}-\frac{1}{\theta}\right) \lambda \int_{\mathbb{R}^{N}} W(x)\left|G^{-1}\left(v_{n}\right)\right|^{p} d x \\
\geq & \left(\frac{1}{2}-\frac{1}{\theta}\right) \int_{\mathbb{R}^{N}}|\nabla v|^{2} d x+\left(\frac{1}{2}-\frac{1}{\theta}\right) \int_{\mathbb{R}^{N}} V(x)\left|G^{-1}\left(v_{n}\right)\right|^{2} d x \\
& +\int_{\mathbb{R}^{N}} K(x)\left[\frac { 1 } { \theta } h \left(G^{-1}\left(v_{n}\right) G^{-1}\left(v_{n}-H\left(G^{-1}\left(v_{n}\right)\right)\right] d x\right.\right. \\
\geq & \left(\frac{1}{2}-\frac{1}{\theta}\right) \int_{\mathbb{R}^{N}}|\nabla v|^{2} d x+\left(\frac{1}{2}-\frac{1}{\theta}\right) \int_{\mathbb{R}^{N}} V(x)\left|v_{n}\right|^{2} d x .
\end{aligned}
$$


By the Hölder inequality, we have

$$
\int_{\mathbb{R}^{N}} W(x)\left|G^{-1}\left(v_{n}\right)\right|^{p} d x \leq \frac{1}{a^{p}} \int_{\mathbb{R}^{N}} W(x)\left|v_{n}\right|^{p} d x \leq\|W(x)\|_{\frac{2}{2-p}}\|v\|^{p} .
$$

Therefore,

$$
c+1+o_{n}(1)\left\|v_{n}\right\|+\left(\frac{1}{p}-\frac{1}{\theta}\right) \lambda\|W(x)\|_{\frac{2}{2-p}}\|v\|^{p} \geq\left(\frac{1}{2}-\frac{1}{\theta}\right)\left\|v_{n}\right\|^{2} .
$$

Then $\left\{v_{n}\right\}$ is bounded in $H$.

\section{Denote}

$$
\begin{array}{r}
J(v)=\frac{1}{2} \int_{\mathbb{R}^{N}}|\nabla v|^{2} d x+\frac{1}{2} \int_{\mathbb{R}^{N}} V(x)|v|^{2} d x-\frac{\lambda}{p} \int_{\mathbb{R}^{N}} W(x)\left|G^{-1}\left(v_{n}\right)\right|^{p} d x \\
-\int_{\mathbb{R}^{N}} K(x) F(x, v) d x,
\end{array}
$$

where

$$
F(x, v)=\int_{0}^{v} f(x, s) d s=\frac{1}{2} \frac{V(x)}{K(x)} v^{2}-\frac{1}{2} \frac{V(x)}{K(x)}\left|G^{-1}(v)\right|^{2}+H\left(G^{-1}(v)\right) .
$$

Taking the derivative, we have

$$
\frac{d}{d t} F(x, t)=f(x, t)=\frac{V(x)}{K(x)} t-\frac{V(x)}{K(x)} \frac{G^{-1}(t)}{g\left(G^{-1}(t)\right)}+\frac{h\left(G^{-1}(t)\right)}{g\left(G^{-1}(t)\right)} .
$$

Moreover, we have the following properties:

Lemma 3.3. Suppose that $h$ satisfies $(\mathrm{h} 2)$ and $(V, K) \in \mathcal{K}$, then
(1) $\lim _{t \rightarrow 0} \frac{f(x, t)}{t}=0$.
(2) $\lim _{t \rightarrow+\infty} \frac{f(x, t)}{t^{2^{*}-1}}=0$. 
Proof. Since $h$ satisfies (h2), combining (4) of Lemma 2.1, by a direct calculation, we get

(1)

$$
\begin{aligned}
\lim _{t \rightarrow 0} \frac{f(x, t)}{t} & =\lim _{t \rightarrow 0}\left[\frac{V(x)}{K(x)}-\frac{V(x)}{K(x)} \frac{G^{-1}(t)}{\operatorname{tg}\left(G^{-1}(t)\right)}+\frac{h\left(G^{-1}(t)\right)}{\operatorname{tg}\left(G^{-1}(t)\right)}\right] \\
& =\frac{V(x)}{K(x)}-\lim _{t \rightarrow 0} \frac{V(x)}{K(x)} \frac{G^{-1}(t)}{\operatorname{tg}\left(G^{-1}(t)\right)}+\lim _{t \rightarrow 0} \frac{h\left(G^{-1}(t)\right)}{G^{-1}(t)} \frac{G^{-1}(t)}{\operatorname{tg}\left(G^{-1}(t)\right)} \\
& =0 .
\end{aligned}
$$

$$
\begin{aligned}
& \lim _{t \rightarrow+\infty} \frac{f(x, t)}{t^{2^{*}-1}} \\
& =\lim _{t \rightarrow+\infty}\left[\frac{V(x)}{K(x) t^{2^{*}-2}}-\frac{V(x)}{K(x)} \frac{G^{-1}(t)}{t^{2^{*}-1} g\left(G^{-1}(t)\right)}+\frac{h\left(G^{-1}(t)\right)}{t^{2^{*}-1} g\left(G^{-1}(t)\right)}\right] \\
& =\lim _{t \rightarrow+\infty}\left[\frac{V(x)}{K(x) t^{2^{*}-2}}-\frac{V(x)}{K(x)} \frac{G^{-1}(t)}{t} \frac{1}{t^{2^{*}-2} g\left(G^{-1}(t)\right)}+\frac{h\left(G^{-1}(t)\right)}{\left(G^{-1}(t)\right)^{2^{*}-1}} \frac{\left(G^{-1}(t)\right)^{2^{*}-1}}{t^{2^{*}-1} g\left(G^{-1}(t)\right)}\right] \\
& =0 .
\end{aligned}
$$

This completes the proof.

Lemma 3.4. Assume that $h$ satisfies (h2) and $(V, K) \in \mathcal{K}$, let $\left\{v_{n}\right\} \subset H$ be a bounded $(P S)_{c}$ sequence and $v_{n} \rightarrow v, v \in H$, then

$$
\lim _{n \rightarrow \infty} \int_{\mathbb{R}^{N}} K(x) f\left(x, v_{n}\right) v_{n} d x=\int_{\mathbb{R}^{N}} K(x) f(x, v) v d x
$$

and

$$
\lim _{n \rightarrow \infty} \int_{\mathbb{R}^{N}} K(x) f\left(x, v_{n}\right) v d x=\int_{\mathbb{R}^{N}} K(x) f(x, v) v d x
$$


Proof. It deduces from Lemma 3.3 and (h2), $f$ satisfies all conditions of Lemma 1.1 in [3], we obtain the conclusion.

Lemma 3.5. Assume that $W \in L^{\frac{2}{2-p}}\left(\mathbb{R}^{N}, \mathbb{R}^{+}\right), 1<p<2$, let $\left\{v_{n}\right\} \subset H$ be a bounded $(P S)_{c}$ sequence and $v_{n} \rightarrow v, v \in H$, then

$$
\lim _{n \rightarrow \infty} \int_{\mathbb{R}^{N}} W(x) \frac{\left|G^{-1}\left(v_{n}\right)\right|^{p-2} G^{-1}\left(v_{n}\right)}{g\left(G^{-1}\left(v_{n}\right)\right)} v_{n} d x=\int_{\mathbb{R}^{N}} W(x) \frac{\left|G^{-1}(v)\right|^{p-2} G^{-1}(v)}{g\left(G^{-1}(v)\right)} v d x
$$

and

$$
\lim _{n \rightarrow \infty} \int_{\mathbb{R}^{N}} W(x) \frac{\left|G^{-1}\left(v_{n}\right)\right|^{p-2} G^{-1}\left(v_{n}\right)}{g\left(G^{-1}\left(v_{n}\right)\right)} v d x=\int_{\mathbb{R}^{N}} W(x) \frac{\left|G^{-1}(v)\right|^{p-2} G^{-1}(v)}{g\left(G^{-1}(v)\right)} v d x .
$$

Proof. Let $\xi(t)=\frac{\left|G^{-1}(t)\right|^{p-2} G^{-1}(t)}{g\left(G^{-1}(t)\right)} t$, since $G^{-1}$ is odd and $G^{-1}(t) \geq 0$

when $t \geq 0$, by (3) and (5) of Lemma 2.1, we know that

$$
0 \leq \xi(t) \leq \frac{1}{a^{p}}|t|^{p}
$$

By Lemma 2.7 of [56], we have

$$
\lim _{n \rightarrow \infty} \int_{\mathbb{R}^{N}} W(x)\left|v_{n}-v\right|^{p} d x=0
$$

Hence,

$$
\lim _{n \rightarrow \infty} \int_{\mathbb{R}^{N}} W(x) \xi\left(v_{n}-v\right) \leq \frac{1}{a^{p}} \lim _{n \rightarrow \infty} \int_{\mathbb{R}^{N}} W(x)\left|v_{n}-v\right|^{p} d x=0 .
$$

This completes the proof.

Third, we prove $J$ satisfies $(P S)_{c}$ condition. 
Lemma 3.6. Suppose that $h$ satisfies $(\mathrm{h} 2)$ and $(V, K) \in \mathcal{K}$, then any $(P S)_{c}$ sequence $\left\{v_{n}\right\} \subset H$ is obtained in (3.2) has a strong convergence subsequence.

Proof. By Lemma 3.2, $\left\{v_{n}\right\}$ in bounded in $H$, up to a subsequence, denote as $\left\{v_{n}\right\}$. Assume that $v_{n} \rightarrow v, v \in H$, since $\left\langle J_{\kappa, \lambda}^{\prime}\left(v_{n}\right), v_{n}\right\rangle=o_{n}(1)$, together with Lemma 3.4, we have

$$
\begin{aligned}
\lim _{n \rightarrow \infty}\left\|v_{n}\right\|^{2} & =\lim _{n \rightarrow \infty} \int_{\mathbb{R}^{N}} K(x) f\left(x, v_{n}\right) v_{n} d x+\lim _{n \rightarrow \infty} \int_{\mathbb{R}^{N}} W(x) \frac{\left|G^{-1}\left(v_{n}\right)\right|^{p-2} G^{-1}\left(v_{n}\right)}{g\left(G^{-1}\left(v_{n}\right)\right)} v_{n} d x \\
& =\int_{\mathbb{R}^{N}} K(x) f(x, v) v d x+\int_{\mathbb{R}^{N}} W(x) \frac{\left|G^{-1}(v)\right|^{p-2} G^{-1}(v)}{g\left(G^{-1}(v)\right)} v d x .
\end{aligned}
$$

Moreover, it deduces from $\left\langle J^{\prime}\left(v_{n}\right), v\right\rangle=o_{n}(1)$ that

$$
\left\langle v_{n}, v\right\rangle=\int_{\mathbb{R}^{N}} K(x) f\left(x, v_{n}\right) v d x+\int_{\mathbb{R}^{N}} W(x) \frac{\left|G^{-1}\left(v_{n}\right)\right|^{p-2} G^{-1}\left(v_{n}\right)}{g\left(G^{-1}\left(v_{n}\right)\right)} v d x+o_{n}(1) .
$$

Then

$$
\begin{aligned}
\lim _{n \rightarrow \infty}\left\langle v_{n}, v\right\rangle & =\lim _{n \rightarrow \infty} \int_{\mathbb{R}^{N}} K(x) f\left(x, v_{n}\right) v d x+\int_{\mathbb{R}^{N}} W(x) \frac{\left|G^{-1}\left(v_{n}\right)\right|^{p-2} G^{-1}\left(v_{n}\right)}{g\left(G^{-1}\left(v_{n}\right)\right)} v d x \\
& =\int_{\mathbb{R}^{N}} K(x) f(x, v) v d x+\int_{\mathbb{R}^{N}} W(x) \frac{\left|G^{-1}(v)\right|^{p-2} G^{-1}(v)}{g\left(G^{-1}(v)\right)} v d x \\
& =\langle v, v\rangle,
\end{aligned}
$$

that is,

$$
\lim _{n \rightarrow \infty}\left\|v_{n}\right\|^{2}=\|v\|^{2}
$$

Therefore, $v_{n} \rightarrow v$ in $H$. 
Proof of Theorem 1.1. By Lemma 3.1 and the Mountain Pass Lemma, for each $\lambda \in\left(0, \lambda_{0}\right)$, there exists a $(P S)_{c}$ sequence $\left\{v_{n}\right\} \subset H$ for $J$. By Lemma 3.2, $\left\{v_{n}\right\}$ is bounded in $H$. Moreover, by Lemma 3.6, there are a subsequence of $\left\{v_{n}\right\}$ and $v_{1} \in H$ such that $v_{n} \rightarrow v_{1}$ with $J\left(v_{1}\right)=c \geq \rho>0$.

Next, we state the second nontrivial solution of problem (2.1) through the local minimization. Since $W \in L^{\frac{2}{2-p}}\left(\mathbb{R}^{N}, \mathbb{R}^{+}\right)$, we choose a function $\phi \in H$ such that

$$
\int_{\mathbb{R}^{N}} W(x)|\phi|^{p} d x>0 .
$$

Thus, by (h3) and Lemma 2.1(3), we have

$$
\begin{aligned}
J(t \phi)= & \frac{t^{2}}{2} \int_{\mathbb{R}^{N}}|\nabla \phi|^{2} d x+\frac{1}{2} \int_{\mathbb{R}^{N}} V(x)\left|G^{-1}(t \phi)\right|^{2} d x-\int_{\mathbb{R}^{N}} K(x) H\left(G^{-1}(t \phi)\right) d x \\
& -\frac{\lambda}{p} \int_{\mathbb{R}^{N}} W(x)\left|G^{-1}(t \phi)\right|^{p} d x \\
\leq & \frac{t^{2}}{2} \int_{\mathbb{R}^{N}}|\nabla \phi|^{2} d x+\frac{t^{2}}{2 a^{2}} \int_{\mathbb{R}^{N}} V(x)|\phi|^{2} d x-\frac{\lambda}{p} t^{p} \int_{\mathbb{R}^{N}} W(x)|\phi|^{p} d x \\
\leq & \frac{t^{2}}{2 a^{2}}\|\phi\|^{2}-\frac{\lambda}{p} t^{p} \int_{\mathbb{R}^{N}} W(x)|\phi|^{p} d x \\
< & 0,
\end{aligned}
$$

for $t>0$ is small enough. Then, there exists $\rho>0$ is given in Lemma 3.1 such that $-\infty<\inf _{v \in B_{\rho}} J(v)<0$. By the Ekeland's variational principle, there exists a minimizing sequence $\left\{v_{n}\right\} \subset \bar{B}_{\rho}$ such that $J\left(v_{n}\right) \rightarrow \inf _{v \in \bar{B}_{\rho}} J(v)$, 
$J^{\prime}\left(v_{n}\right) \rightarrow 0$ as $n \rightarrow \infty$. Hence, by Lemma 3.6, there exists a nontrivial solution $v_{2}$ of problem (2.1) satisfies

$$
J\left(v_{2}\right)<0 \text { and }\left\|v_{2}\right\| \leq \rho .
$$

Therefore, we can conclude that

$$
J\left(v_{2}\right)<0<J\left(v_{1}\right),
$$

for all $\lambda \in\left(0, \lambda_{0}\right)$.

Proof of Theorem 1.2. Since $H$ is a separable Hilbert space, $H$ has a countable orthogonal basis $\left\{e_{i}\right\}, i=1,2, \ldots$ Let $V_{k}=\operatorname{span}\left\{e_{1}, e_{2}, \cdots, e_{k}\right\}$ and $E_{k}=V_{k}^{\perp}$, then $H=V_{k} \oplus E_{k}, k \in \mathbb{N}$. Hence, by Lemma 3.1, we have

$$
\left.J\right|_{S_{\rho} \cap E_{k}} \geq \beta>0
$$

Indeed, for any finite-dimensional subspace $\tilde{H} \subset H$, there is a positive integral number $k$ such that $\widetilde{H} \subset V_{k}$. Suppose to the contrary that there exists a sequence $\left\{v_{n}\right\}$ such that $\left\{v_{n}\right\} \subset \widetilde{H} \subset V_{k}$ and $\left\|v_{n}\right\| \rightarrow \infty$ as $n \rightarrow \infty$, but

$$
\begin{array}{rl}
\frac{1}{2} \int_{\mathbb{R}^{N}}\left|\nabla v_{n}\right|^{2} d x+\frac{1}{2} \int_{\mathbb{R}^{N}} V(x)\left|G^{-1}\left(v_{n}\right)\right|^{2} d x>\int_{\mathbb{R}^{N}} & K(x) H\left(G^{-1}\left(v_{n}\right)\right) d x \\
& +\frac{\lambda}{p} \int_{\mathbb{R}^{N}} W(x)\left|G^{-1}\left(v_{n}\right)\right|^{p} d x .
\end{array}
$$

By Lemma 2.1(3), we have

$$
\left[\int_{\mathbb{R}^{N}} K(x) H\left(G^{-1}\left(v_{n}\right)\right) d x+\frac{\lambda}{p} \int_{\mathbb{R}^{N}} W(x)\left|G^{-1}\left(v_{n}\right)\right|^{p} d x\right] /\left\|v_{n}\right\|^{2}<\frac{1}{2 a^{2}} .
$$


Setting $\eta_{n}=v_{n} /\left\|v_{n}\right\|$, then, $\left\{\eta_{n}\right\}$ is bounded in $H$. Up to a subsequence, we assume that $\eta_{n} \rightarrow \eta$ in $H, \eta_{n} \rightarrow \eta$ in $L^{r}\left(\mathbb{R}^{N}\right)$, $r \in\left[2,2^{*}\right)$ and $\eta_{n}(x) \rightarrow \eta(x)$ a.e. $x \in \mathbb{R}^{N}$. Let $\Omega_{1}=\left\{x \in \mathbb{R}^{N}: \eta(x) \neq 0\right\}$ and $\Omega_{2}=\left\{x \in \mathbb{R}^{N}: \eta(x)=0\right\}$. If meas $\left(\Omega_{1}\right)>0$, by (3.1) and the Fatou's Lemma, we have

$$
\begin{aligned}
& \frac{\int_{\mathbb{R}^{N}} K(x) H\left(G^{-1}\left(v_{n}\right)\right) d x}{\left\|v_{n}\right\|^{2}}=\int_{\mathbb{R}^{N}} \frac{K(x) H\left(G^{-1}\left(v_{n}\right)\right)}{\left|G^{-1}\left(v_{n}\right)\right|^{2}} \frac{\left|G^{-1}\left(v_{n}\right)\right|^{2}}{\left|v_{n}\right|^{2}} \eta_{n}^{2} d x \rightarrow+\infty \\
& \text { as } n \rightarrow \infty \text {. }
\end{aligned}
$$

Note that $1<p<2$, we know that

$$
\begin{aligned}
& \frac{\frac{\lambda}{p} \int_{\mathbb{R}^{N}} W(x)\left|G^{-1}\left(v_{n}\right)\right|^{p} d x}{\left\|v_{n}\right\|^{2}} \geq \frac{\lambda}{p} \int_{\mathbb{R}^{N}} W(x) \frac{\left|v_{n}\right|^{p}}{\left\|v_{n}\right\|^{2}} d x=\frac{\lambda}{p} \int_{\mathbb{R}^{N}} W(x) \frac{\left|\eta_{n}\right|^{p}}{\left\|v_{n}\right\|^{2-p}} d x \rightarrow 0 \\
& \text { as } n \rightarrow \infty \text {. }
\end{aligned}
$$

Which is a contradiction with (3.3). Hence, $\eta_{n} \rightarrow 0$ in $L^{r}\left(\mathbb{R}^{N}\right)$. Since all norms are equivalent on the finite dimensional space, there exists a constant $C>0$ such that

$$
\|u\|_{r} \geq C\|u\|, \forall u \in \widetilde{H}
$$

Hence,

$$
0=\lim _{n \rightarrow \infty}\left\|\eta_{n}\right\|_{r} \geq C \lim _{n \rightarrow \infty}\left\|\eta_{n}\right\|=C>0 .
$$

This is a contradiction and the desired conclusion is obtained. This means that there is a constant $R=R(\tilde{H})>0$ such that $J<0$ on $\tilde{H} \backslash B_{R(\tilde{H})}$. Moreover, by conditions (h1) and (h4), we know that $J(0)=0$ and $J$ is even. Thereby, it follows from Theorem 9.12 in [39], we complete the proof. 


\section{Acknowledgements}

This work was supported by the National Natural Science Foundation of China (No. 11901345), the Yunnan Local Colleges Applied Basic Research Projects (Nos. 2017FH001-011, 2019010070143).

\section{References}

[1] José F. L. Aires and Marco A. S. Souto, Existence of solutions for a quasilinear Schrödinger equation with vanishing potentials, Journal of Mathematical Analysis and Applications 416(2) (2014), 924-946.

DOI: https://doi.org/10.1016/j.jmaa.2014.03.018

[2] José F. L. Aires and Marco A. S. Souto, Equation with positive coefficient in the quasilinear term and vanishing potential, Topological Methods in Nonlinear Analysis 46(2) (2015), 813-833.

DOI: https://doi.org/10.12775/TMNA.2015.069

[3] C. O. Alves and Marco A. S. Souto, Existence of solutions for a class of nonlinear Schrödinger equations with potential vanishing at infinity, Journal of Differential Equations 254(4) (2013), 1977-1991.

DOI: https://doi.org/10.1016/j.jde.2012.11.013

[4] C. O. Alves, Y. J. Wang and Y. T. Shen, Soliton solutions for a class of quasilinear Schrödinger equations with a parameter, Journal of Differential Equations 259(1) (2015), 318-343.

DOI: https://doi.org/10.1016/j.jde.2015.02.030

[5] A. Ambrosetti, V. Felli and A. Malchiodi, Ground states of nonlinear Schrödinger equations with potentials vanishing at infinity, Journal of the European Mathematical Society 7(1) (2005), 117-144.

DOI: https://doi.org/10.4171/JEMS/24

[6] A. Ambrosetti, A. Malchiodi and D. Ruiz, Bound states of nonlinear Schrödinger equations with potentials vanishing at infinity, Journal d'Analyse Mathématique 98(1) (2006), 317-348.

DOI: https://doi.org/10.1007/BF02790279

[7] A. Ambrosetti and Z. Q. Wang, Nonlinear Schrödinger equations with vanishing and decaying potentials, Differential Integral Equations 18(12) (2005), 1321-1332.

[8] H. Berestycki and P. L. Lions, Nonlinear scalar field equations I: Existence of a ground state, Archive for Rational Mechanics and Analysis 82(4) (1983), 313-345.

DOI: https://doi.org/10.1007/BF00250555 
[9] L. Brizhik, A. Eremko, B. Piette and W. J. Zakrzewski, Electron self-trapping in a discrete two-dimensional lattice, Physica D: Nonlinear Phenomena 159(1-2) (2001), 71-90.

DOI: https://doi.org/10.1016/S0167-2789(01)00332-3

[10] D. Bonheure and J. V. Schaftingen, Groundstates for the nonlinear Schrödinger equation with potential vanishing at infinity, Annali di Matematica Pura ed Applicata 189(2) (2010), 273-301.

DOI: https://doi.org/10.1007/s10231-009-0109-6

[11] A. de Bouard and N. Hayashi and J. C. Saut, Global existence of small solutions to a relativistic nonlinear Schrödinger equation, Communications in Mathematical Physics 189(1) (1997), 73-105.

DOI: https://doi.org/10.1007/s002200050191

[12] S. T. Chen and Z. Gao, An improved result on ground state solutions of quasilinear Schrödinger equations with super-linear nonlinearities, Bulletin of the Australian Mathematical Society 99(2) (2019), 231-241.

DOI: https://doi.org/10.1017/S0004972718001235

[13] B. T. Cheng and X. H. Tang, High energy solutions of modified quasilinear fourthorder elliptic equations with sign-changing potential, Computers \& Mathematics with Applications 73(1) (2017), 27-36.

DOI: https://doi.org/10.1016/j.camwa.2016.10.015

[14] Y. K. Cheng and Y. X. Yao, Soliton solutions to a class of relativistic nonlinear Schrödinger equations, Applied Mathematics and Computation 260 (2015), 342-350.

DOI: https://doi.org/10.1016/j.amc.2015.03.055

[15] Y. K. Cheng and J. Yang, The existence and uniqueness result for a relativistic nonlinear Schrödinger equation, Abstract and Applied Analysis (2014); Article ID 362985, 1-10.

DOI: https://doi.org/10.1155/2014/362985

[16] C. M. Chu and H. D. Liu, Existence of positive solutions for a quasilinear Schrödinger equation, Nonlinear Analysis: Real World Applications 44 (2018), 118-127.

DOI: https://doi.org/10.1016/j.nonrwa.2018.04.007

[17] M. Colin and L. Jeanjean, Solutions for a quasilinear Schrödinger equation: A dual approach, Nonlinear Analysis: Theory, Methods \& Applications 56(2) (2004), 213-226.

DOI: https://doi.org/10.1016/j.na.2003.09.008 
[18] Y. B. Deng and W. Shuai, Non-trivial solutions for a semilinear biharmonic problem with critical growth and potential vanishing at infinity, Proceedings of the Royal Society of Edinburgh Section A: Mathematics 145(2) (2015), 281-299.

DOI: https://doi.org/10.1017/S0308210513001170

[19] Y. B. Deng and W. Shuai, Positive solutions for quasilinear Schrödinger equations with critical growth and potential vanishing at infinity, Communications on Pure \& Applied Analysis 13(6) (2014), 2273-2287.

DOI: https://doi.org/10.3934/cpaa.2014.13.2273

[20] J. M. do Ó, E. Gloss and C. Santana, Solitary waves for a class of quasilinear Schrödinger equations involving vanishing potentials, Advanced Nonlinear Studies 15(3) (2015), 691-714.

DOI: https://doi.org/10.1515/ans-2015-0308

[21] H. Hartmann and W. J. Zakrzewski, Electrons on hexagonal lattices and applications to nanotubes, Physical Review B 68(18) (2003), 184-302.

DOI: https://doi.org/10.1103/PhysRevB.68.184302

[22] C. Huang and G. Jia, Existence of positive solutions for supercritical quasilinear Schrödinger elliptic equations, Journal of Mathematical Analysis and Applications 472(1) (2019), 705-727.

DOI: https://doi.org/10.1016/j.jmaa.2018.11.048

[23] L. Jeanjean and K. Tanaka, A positive solution for a nonlinear Schrödinger equation on $\mathbb{R}^{N}$, Indiana University Mathematics Journal 54(2) (2005), 443-464.

DOI: https://doi.org/10.1512/iumj.2005.54.2502

[24] O. Kwon, Existence of standing waves of nonlinear Schrödinger equations with potentials vanishing at infinity, Journal of Mathematical Analysis and Applications 387(2) (2012), 920-930.

DOI: https://doi.org/10.1016/j.jmaa.2011.09.050

[25] G. F. Li, Y. S Huang and Z. Liu, Positive solutions for quasilinear Schrödinger equations with superlinear term, Complex Variables and Elliptic Equations 65(6) (2019), 936-955.

DOI: https://doi.org/10.1080/17476933.2019.1636791

[26] G. F. Li and Y. S Huang, Positive solutions for generalized quasilinear Schrödinger equations with asymptotically linear nonlinearities, Applicable Analysis (2019).

DOI: https://doi.org/10.1080/00036811.2019.1634256

[27] J. Q. Liu, Y. Q. Wang and Z. Q. Wang, Solutions for quasilinear Schrödinger equations via the Nehari method, Communications in Partial Differential Equations 29(5-6) (2004), 879-901.

DOI: https://doi.org/10.1081/PDE-120037335 
[28] Z. P. Liang, J. F. Gao and A. R. Li, Infinitely many solutions to a quasilinear Schrödinger equation with a local sublinear term, Applied Mathematics Letters 89 (2019), 22-27.

DOI: https://doi.org/10.1016/j.aml.2018.09.015

[29] Q. Q. Li, K. M. Teng and X. Wu, Existence of positive solutions for a class of critical fractional Schrödinger equations with potential vanishing at infinity, Mediterranean Journal of Mathematics 14(2) (2017); Article 80.

DOI: https://doi.org/10.1007/s00009-017-0846-5

[30] Q. Q. Li and X. Wu, Existence, multiplicity, and concentration of solutions for generalized quasilinear Schrödinger equations with critical growth, Journal of Mathematical Physics 58(4) (2017); Article 041501.

DOI: https://doi.org/10.1063/1.4982035

[31] Q. Q. Li and X. Wu, Multiple solutions for generalized quasilinear Schrödinger equations, Mathematical Methods in the Applied Sciences 40(5) (2017), 1359-1366.

$$
\text { DOI: https://doi.org/10.1002/mma.4050 }
$$

[32a] P. L. Lions, The concentration-compactness principle in the calculus of variation, The locally compact case: Part I, Annales de l'I.H.P: Analyse Non Linéaire 1(2) (1984), 109-145.

[32b] P. L. Lions, The concentration-compactness principle in the calculus of variation, The locally compact case: Part II, Annales de l'I.H.P: Analyse Non Linéaire 1(4) (1984), 223-283.

[33] X. Q. Liu, J. Q. Liu and Z. Q. Wang, Quasilinear elliptic equations via perturbation method, Proceedings of the American Mathematical Society 141(1) (2013), 253-263.

DOI: https://doi.org/10.1090/S0002-9939-2012-11293-6

[34] J. Q. Liu, X. Q. Liu and Z. Q. Wang, Multiple sign-changing solutions for quasilinear elliptic equations via perturbation method, Communications in Partial Differential Equations 39(12) (2014), 2216-2239.

DOI: https://doi.org/10.1080/03605302.2014.942738

[35] X. Q. Liu, J. Q. Liu and Z. Q. Wang, Quasilinear elliptic equations with critical growth via perturbation method, Journal of Differential Equations 254(1) (2013), 102-124.

DOI: https://doi.org/10.1016/j.jde.2012.09.006

[36] X. N. Liu and H. B. Chen, Positive solutions for a class of quasilinear Schrödinger equations with vanishing potentials, Boundary Value Problems (2017); Article ID 35,11 pages.

DOI: https://doi.org/10.1186/s13661-017-0769-x 
[37] V. F. Morales Delgado, J. F. Gómez Aguilar and D. Baleanu, A new approach to exact optical soliton solutions for the nonlinear Schrödinger equation, European Physical Journal Plus 133(5) (2018); Article ID 189.

DOI: https://doi.org/10.1140/epjp/i2018-12020-4

[38] M. Poppenberg, K. Schmitt and Z. Q. Wang, On the existence of soliton solutions to quasilinear Schrödinger equations, Calculus of Variations and Partial Differential Equations 14(3) (2002), 329-344.

DOI: https://doi.org/10.1007/s005260100105

[39] P. H. Rabinowitz, in: Minimax Methods in Critical Point Theory with Applications to Differential Equations, in: CBMS Regional Conference Series in Mathematics, Volume 65, American Mathematical Society, Providence RI, 1986.

[40] D. Ruiz and G. Siciliano, Existence of ground states for a modified nonlinear Schrödinger equation, Nonlinearity 23(5) (2010), 1221-1233.

DOI: https://doi.org/10.1088/0951-7715/23/5/011

[41] U. B. Severo, E. Gloss and E. D. da Silva, On a class of quasilinear Schrödinger equations with superlinear or asymptotically linear terms, Journal of Differential Equations 263(6) (2017), 3550-3580.

DOI: https://doi.org/10.1016/j.jde.2017.04.040

[42] U. B. Severo and M. Gilson de Carvalho, Quasilinear Schrödinger equations with unbounded or decaying potentials, Mathematische Nachrichten 291(2-3) (2018), 492-517.

DOI: https://doi.org/10.1002/mana.201600028

[43] U. B. Severo and G. M. de Carvalho, Quasilinear Schrödinger equations with a positive parameter and involving unbounded or decaying potentials, Applicable Analysis (2019).

DOI: https://doi.org/10.1080/00036811.2019.1599106

[44] Y. T. Shen and Y. J. Wang, Standing waves for a class of quasilinear Schrödinger equations, Complex Variables and Elliptic Equations 61(6) (2016), 817-842.

DOI: https://doi.org/10.1080/17476933.2015.1119818

[45] Y. T. Shen and Y. J. Wang, Soliton solutions for generalized quasilinear Schrödinger equations, Nonlinear Analysis: Theory, Methods \& Applications 80 (2013), 194-201.

DOI: https://doi.org/10.1016/j.na.2012.10.005

[46] Y. T. Shen and Y. J. Wang, Standing waves for a relativistic quasilinear asymptotically Schrödinger equation, Applicable Analysis 95(11) (2016), 2553-2564.

DOI: https://doi.org/10.1080/00036811.2015.1100296 
[47] Y. T. Shen and Y. J. Wang, A class of generalized quasilinear Schrödinger equations, Communications on Pure \& Applied Analysis 15(3) (2016), 853-870.

DOI: https://doi.org/10.3934/cpaa.2016.15.853

[48] H. X. Shi and H. B. Chen, Positive solutions for generalized quasilinear Schrödinger equations with potential vanishing at infinity, Applied Mathematics Letters 61 (2016), 137-142.

DOI: https://doi.org/10.1016/j.aml.2016.06.004

[49] H. X. Shi and H. B. Chen, Existence and multiplicity of solutions for a class of generalized quasilinear Schrödinger equations, Journal of Mathematical Analysis and Applications 452(1) (2017), 578-594.

DOI: https://doi.org/10.1016/j.jmaa.2017.03.020

[50] H. X. Shi and H. B. Chen, Infinitely many solutions for generalized quasilinear Schrödinger equations with a finite potential well, Bulletin of the Iranian Mathematical Society 44(3) (2018), 691-705.

DOI: https://doi.org/10.1007/s41980-018-0044-7

[51] Elves A. B. Silva and Gilberto F. Vieira, Quasilinear asymptotically periodic Schrödinger equations with critical growth, Calculus of Variations and Partial Differential Equations 39(1) (2010), 1-33.

DOI: https://doi.org/10.1007/s00526-009-0299-1

[52] Y. J. Wang, Solitary solutions for a class of Schrödinger equations in $\mathbb{R}^{3}$, Zeitschrift für angewandte Mathematik und Physik 67(4) (2016); Article ID 88, 17 pages.

DOI: https://doi.org/10.1007/s00033-016-0679-2

[53] Y. J. Wang, A class of quasilinear Schrödinger equations with critical or supercritical exponents, Computers \& Mathematics with Applications 70(4) (2015), 562-572.

DOI: https://doi.org/10.1016/j.camwa.2015.05.016

[54] X. Y. Yang, W. B. Wang and F. K. Zhao, Infinitely many radial and non-radial solutions to a quasilinear Schrödinger equation, Nonlinear Analysis: Theory, Methods \& Applications 114 (2015), 158-168.

DOI: https://doi.org/10.1016/j.na.2014.11.015

[55] Z. P. Yang and F. K. Zhao, Three solutions for a fractional Schrödinger equation with vanishing potentials, Applied Mathematics Letters 76 (2018), 90-95.

DOI: https://doi.org/10.1016/j.aml.2017.08.004

[56] W. Zhang, J. Zhang and Z. M. Luo, Multiple solutions for the fourth-order elliptic equation with vanishing potential, Applied Mathematics Letters 73 (2017), 98-105.

DOI: https://doi.org/10.1016/j.aml.2017.04.030 